\title{
Análise de Estabilidade de Tensão de Sistemas Elétricos Usando uma Rede Neural Artmap Fuzzy
}

\author{
L.Y. ISODA ${ }^{1}$, A.D.P. LOTUFO ${ }^{2}$ M.L.M. LOPES, ${ }^{3}$ C.R. MINUSSI ${ }^{4}$ FEIS, UNESP, \\ Campus de Ilha Solteira, 15385-000 Ilha Solteira, SP, Brasil.
}

\begin{abstract}
Resumo. Apresenta-se, neste trabalho, uma nova metodologia para o diagnóstico da estabilidade de tensão estática de sistemas elétricos de potência. Esta metodologia refere-se a um sistema neural de inferência baseado em uma arquitetura neural ARTMAP fuzzy, cujo treinamento é realizado a partir de uma base de dados gerada, via simulação, usando-se um programa computacional: cálculo de fluxo de potência, margem de segurança e montagem da base de dados (que constitui os estímulos de entrada / saída da rede neural). Este sistema se destaca por apresentar resultados precisos com alta rapidez de resposta, o que permite, aos usuários, trabalhar com mais flexibilidade em ambiente em que modificações estruturais são requeridas (situação real de operação dos sistemas), se comparadas às demais redes neurais. Como forma de ilustrar a estrutura neural proposta, apresenta-se um aplicação considerando-se um sistema elétrico de potência composto por 45 barras, 72 linhas de transmissão e 10 máquinas síncronas.
\end{abstract}

Palavras-chave. Sistemas elétricos de potência, estabilidade de tensão, teoria da ressonância adaptativa.

\section{Introdução}

A estabilidade de tensão refere-se à investigação sobre o comportamento do perfil de tensão nodal do sistema, em destaque, a observação e identificação da deficiência de suporte de tensão em decorrência do aumento da potência consumida.

A estabilidade de tensão pode ser abordada sob dois pontos de vista: estático e dinâmico. O caráter dinâmico requer um tratamento como um fenômeno que pode ser modelado por um conjunto de equações diferenciais ordinárias não-lineares [8]. Trata-se de uma análise complexa e sofisticada, principalmente quando se aborda sistemas de grande porte. Uma forma mais simples, porém igualmente importante da estabilidade de tensão, refere-se à observação do comportamento das tensões nodais, considerando-se o aumento gradativo do perfil do carregamento do sistema, ou seja, a análise qualitativa do ponto de observação. Neste caso, a análise pode ser tratada como um problema linear. As inferências sobre o sistema são baseadas na análise do comportamento do ponto de equilíbrio, mais especificamente, a partir

\footnotetext{
${ }^{1}$ lilian@mat.feis.unesp.br

2 annadiva@dee.feis.unesp.br

${ }^{3}$ mara@mat.feis.unesp.br

${ }^{4}$ minussi@dee.feis.unesp.br
} 
da análise da matriz jacobiana $\left(J^{0}\right)$ das equações de potência nodais do sistema, constituindo um problema de fluxo de potência, formulado via método de NewtonRaphson $[1,8]$. Deste modo, a partir da análise da matriz jacobiana $J^{0}$, pode-se inferir sobre a estabilidade do sistema. Esta análise pode ser realizada, por exemplo, usando a decomposição de autovalores e de autovetores da matriz jacobiana, vetor tangente, entre outras técnicas. O comportamento do sistema, para pequenas perturbações, pode ser analisado através dos autovalores da matriz $J^{0}$. Se todos os autovalores de $J^{0}$ forem reais e positivos, pode-se concluir que o sistema é estável para pequenas perturbações. Contudo, a instabilidade se caracteriza por haver, pelo menos, um autovalor com a parte real negativa. O ponto limítrofe ocorre quando a matriz jacobiana $J^{0}$ torna-se singular (há a ocorrência de, pelo menos, 1 autovalor nulo). Neste caso, o determinante da matriz jacobiana $J^{0}$ torna-se, também, nulo. Esta tem sido a forma abordada na maioria das referências bibliográficas pertinentes. Neste artigo, propõe-se o desenvolvimento de um sistema neural para execução do diagnóstico da estabilidade (de caráter estático) de tensão de sistemas de energia [1, 8]. O funcionamento das redes neurais é constituído por duas fases fundamentais: treinamento e análise. A fase de treinamento requer uma grande quantidade de processamento para sua realização, enquanto que a fase de análise é efetivada, praticamente, sem esforço computacional. Esta é, portanto, a principal justificativa para o uso de redes neurais para a resolução de problemas complexos que exigem soluções rápidas, como é o caso de aplicações em tempo real. A fase de treinamento da rede neural é realizada, na maioria das vezes, através da técnica de retropropagação [6], que é reconhecidamente muito lenta e, em muitos casos, não converge, principalmente quando se trabalha com grandes bancos de dados. Novas estruturas neurais têm sido abordadas, visando resolver esta questão do excessivo tempo de processamento para a realização do treinamento. Neste contexto, destacam-se as redes neurais da família ART (Adaptive Resonance Theory) [3], que apresentam as características de estabilidade (capacidade de aprender através do ajuste dos seus pesos) e plasticidade (capacidade de continuar a aprender com a inclusão de novos padrões, sem perder a memória relacionada aos padrões anteriores). Assim, neste artigo apresenta-se um sistema neural para a execução do diagnóstico da estabilidade estática de tensão. A rede neural usada é uma arquitetura ARTdescendente [3], ou seja, uma rede ARTMAP fuzzy com inclusão de melhorias, visando torná-la, principalmente, mais precisa, se comparada à formulação original [3]. Esta rede neural possui, como critério de treinamento, um índice (margem) de segurança baseado na análise de sensibilidade da função determinante da matriz $J^{0}$, calculada empregando-se conceitos da álgebra de Kronecker [5]. Visando testar o desempenho da metologia proposta, apresenta-se uma aplicação considerando-se um sistema elétrico de potência composto por 10 barramentos, 72 linhas de transmissão e 10 máquinas síncronas.

\section{Metodologia Proposta}

A seguir, apresenta-se o esquema proposto (sistema neural) para realizar a análise de estabilidade de tensão de sistemas elétricos de potência. O funcionamento das redes 
neurais é basicamente, em duas fases principais: (1) treinamento ou aprendizado; (2) testes e diagnósticos. A rede neural usada é uma arquitetura ARTMAP fuzzy [3]. A fase de treinamento é realizada usando um "professor" representado por um simulador (programa computacional Simul [4] implantado no Laborátorio Computacional do Departamento de Engenharia Elétrica - Unesp - Campus de Ilha Solteira) que realiza cálculo de redes elétricas: matrizes de rede, fluxo de potência e análise de estabilidade transitória. Este programa foi adaptado para executar, também, os cálculos associados à estabilidade estática de tensão, conforme o critério apresentado na Seção 3, e a base de dados, constituída por pares de padrões de entrada e de saída da rede neural, para a realização do treinamento neural. O fluxograma, da referida metodologia, é mostrado na Figura 1.

A parte do fluxograma, com linhas tracejadas, refere-se ao módulo que executa o treinamento continuado. Ou seja, sempre que houver disponibilidade de dados de entrada para a execução da análise de estabilidade de tensão pela rede neural (bloco 1), se julgado como sendo um vetor $(\mathrm{P}, \mathrm{Q}, \mathrm{Z})$ relevante, usa-se este vetor e a saída $(\mathcal{M})$ associada para serem inseridos na base de dados, via adaptação dos pesos. $\mathrm{O}$ vetor $\mathrm{Z}$ contém as informações binárias que codificam a topologia da rede elétrica, os dados das contingências, etc.

\section{Critério de Análise da Estabilidade Estática de Tensão}

A análise de estabilidade estática de sistemas de energia consiste na investigação comportamental do modelo correspondente à solução das equações linearizadas do fluxo de potência [8], executando-se a barra de referência [1]:

$$
\left[\begin{array}{c}
\Delta P \\
\Delta Q
\end{array}\right]=\left[J^{0}\right]\left[\begin{array}{c}
\Delta \theta \\
\Delta V
\end{array}\right]
$$

sendo: $\Delta P$ o vetor das potências ativas das barras $\mathrm{PV}$ e $\mathrm{QP} ; \Delta Q$ o vetor das potências reativas das barras $\mathrm{PQ} ; \Delta \theta$ o vetor dos ângulos nas barras $\mathrm{PV}$ e $\mathrm{PQ} ; \Delta V$ o vetor das tensões nodais das barras PQ e; $J^{0} \Delta$ matriz jacobiana $=\left[\begin{array}{cc}H & N \\ M & L\end{array}\right]$ em que $H, N, M$ e $L$ são as submatrizes de $J^{0}$ correspondente a $\partial P / \partial \theta, \partial P / \partial V$, $\partial Q / \partial Q \theta$ e $\partial Q / \partial V$, respectivamente.

Neste artigo, pretende-se, então, investigar a questão da estabilidade estática de tensão, através da análise de sensibilidade, usando como função o determinante da matriz $J^{0}$. Ressalta-se que o cálculo do determinante de uma matriz pode ser realizado de forma bastante rápida e eficiente [7], se comparado com os principais métodos comumente disponíveis na literatura, ainda que esta função não seja utilizada neste trabalho e sim a sua derivada. Desta forma, através de expansão por série de Taylor, aproximação de primeira ordem, pode-se estimar a variação do determinante da matriz $J^{0}\left(\operatorname{det}\left(J^{0}\right)\right)$, como mostra-se a seguir. 


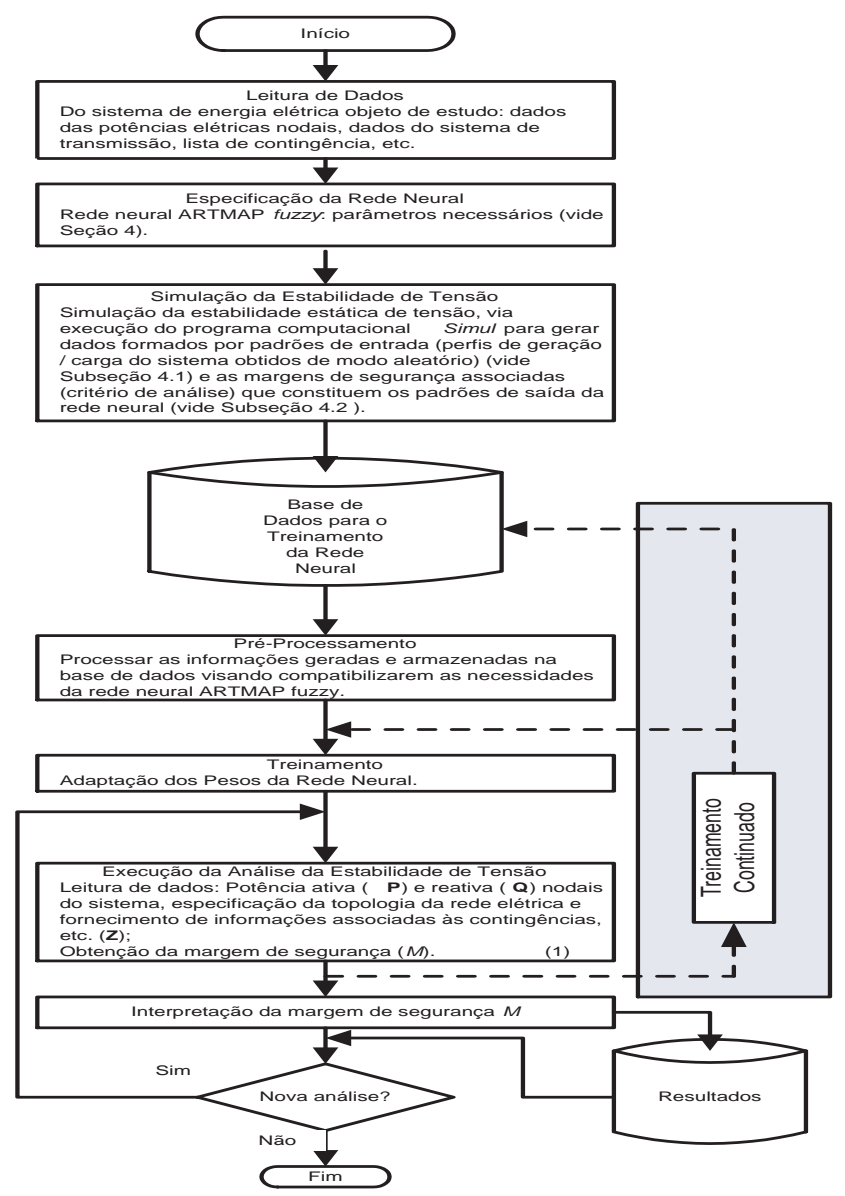

Figura 1: Fluxograma do sistema neural proposto para realizar análise de estabilidade de tensão de sistemas elétricos de potência.

Considere [5]:

$$
\begin{aligned}
f(J) & =f\left(J^{0}\right)+\epsilon \operatorname{Tr}\left\{D\left(J^{0}\right)^{T}\right\}+\phi(\epsilon) \\
& =f\left(J^{0}\right)+\epsilon\left\langle\psi\left(D\left(J^{0}\right)\right), \psi(\Delta J)\right\rangle+\phi(\epsilon), \quad \epsilon \in \mathbb{R} .
\end{aligned}
$$

sendo: $J=J^{0}+\epsilon \Delta J ;\langle u, v\rangle$ o produto interno entre $u$ e $v ; \nabla$ o gradiente de uma função escalar; $D\left(J^{0}\right) \underline{\Delta} \psi^{-1}\left(\nabla_{\psi} f\left(J^{0}\right)\right)=\partial f(J) /\left.\partial J\right|_{0} ; \quad \nabla_{\psi} f\left(J^{0}\right)=\psi\left(D\left(J^{0}\right)\right)$; Tr o traço de uma matriz; ; $\Delta J=\left[\begin{array}{cc}\Delta J_{H} & \Delta J_{N} \\ \Delta J_{M} & \Delta J_{L}\end{array}\right] ; \epsilon=$ parâmetro de perturbação e, $\quad \psi(\Delta J) \underline{\Delta}\left[\Delta J_{11} \ldots \Delta J_{p 1} \ldots \Delta J_{1 p} \ldots \Delta J_{p p}\right]^{T}$.

O determinante da matriz $J$ pode ser expresso por [5]

$$
\left.\operatorname{det}(J)=\operatorname{det}\left\{J^{0}\left(I+\epsilon\left(J^{0}\right)\right)^{-1} \Delta J\right)\right\}, J^{0} \in \mathbb{R}^{p \times p},\left(J^{0}\right)^{-1} \exists
$$


sendo $I$ a matriz identidade $(p \times p)$.

A equação (3.2) pode ser desenvolvida do seguinte modo:

$$
\begin{aligned}
\operatorname{det}(J) & =\operatorname{det}\left(J^{0}\right) \operatorname{det}\left(I+\epsilon\left(J^{0}\right)\right)^{-1} \Delta J \\
& =\operatorname{det}\left(J^{0}\right)+\epsilon \operatorname{Tr}\left(\operatorname{det}\left(J^{0}\right)\left(J^{0}\right)^{-1} \Delta J\right)+\phi(\epsilon)
\end{aligned}
$$

Como $\operatorname{det}\left(J^{0}\right)\left(J^{0}\right)^{-1}=\operatorname{adj}\left(J^{0}\right)$, então, a equação (3.6), utilizando-se as equações (3.1-3.3), pode ser expressa por:

$$
\operatorname{det}(J)=\operatorname{det}\left(J^{0}\right)+\epsilon\left\langle\psi\left(\operatorname{adj}\left(J^{0}\right)^{T}\right), \psi(\Delta J)\right\rangle+\phi(\epsilon)
$$

sendo $\operatorname{adj}\left(J^{0}\right)$ a matriz adjunta de $J^{0}$, i.e., $\operatorname{adj}\left(J^{0}\right)=\operatorname{cof}\left(\left(J^{0}\right)^{T}\right)$.

A partir da equação (3.2), conclui-se que a sensibilidade da função $\operatorname{det}\left(J^{0}\right)$ pode ser expressa por :

$$
\partial \operatorname{det}(J) / \partial J=\psi\left(\operatorname{adj}(J)^{T}\right)
$$

Dividindo-se a equação (3.2) por $\operatorname{det}\left(J^{0}\right)$, obtém-se:

$$
\tau=1+\Delta \tau+\phi(\epsilon)
$$

sendo : $\tau$ a função determinante normalizada, ou seja $\tau \underline{\Delta} \frac{\operatorname{det}(J)}{\operatorname{det}\left(J^{0}\right)}, \quad \psi(\epsilon)=$ $\frac{\phi(\epsilon)}{\operatorname{det}\left(J^{0}\right)}$, e $\Delta \tau=\epsilon\left\langle\psi\left(\left(\left(J^{0}\right)\right)^{-1}\right)^{T}, \psi(\Delta J)\right\rangle$.

Também, a partir da equação (3.4), conclui-se que:

$$
\left(\partial \operatorname{det}\left(J^{0}\right) /\left.\partial J\right|_{J=J^{0}}\right)=\psi\left(\left(\left(J^{0}\right)^{-1}\right)^{T}\right)
$$

A equação (3.5) representa a sensibilidade da função determinante normalizada. Assim, fazendo-se $a=\psi\left\{\left(\left(J^{0}\right)^{-1}\right)^{T}\right\} \quad$ (vetor) e $C \underline{\Delta} \psi\left[\left(J^{0}\right)^{-1}\right]^{T}=\left[\begin{array}{cc}C_{H} & C_{N} \\ C_{M} & C_{L}\end{array}\right]$ obtém-se a seguinte representação para $a$ que é a sensibilidade da função determinante normalizada, na forma vetorial:

$$
a=\left[C_{11} \cdots C_{p 1} \cdots C_{1 p} \cdots C_{p p}\right]^{T} .
$$

A variação linear da função determinante normalizada $\Delta \tau$ pode ser expressa por:

$$
\Delta \tau=\epsilon \sum_{i=1}^{p} \sum_{j=1}^{p} C_{i j} \Delta J_{i j}
$$

Deve-se observar que a matriz $\Delta J$ é uma estrutura esparsa, semelhante à esparsidade da matriz Ybarra. Assim, poucos são os elementos de $\Delta J$ que são diferentes de zero.

Desconsiderando-se os termos não-lineares $\phi(\epsilon)$, a partir da equação (3.4), fazendo-se $\tau=0$ e $\epsilon=1$, obtém-se:

$$
\langle a, \psi(\Delta J)\rangle=-1 .
$$


A equação (3.6) estabelece a relação entre os vetores $\psi\left\{\left(\left(J^{0}\right)^{-1}\right)^{T}\right\}$ e $\psi(\Delta)$ tais que reproduzem, considerando-se o modelo linear, um valor nulo para a função $\operatorname{det}(J)$, ou seja, uma condição limite para a estabilidade de tensão. Deste modo, a partir desta equação, pode-se estimar a distância euclidiana que corresponde à margem de segurança ou a distância em que o ponto de operação do sistema encontra-se em relação à condição limítrofe da estabilidade estática:

$$
\mathcal{M}=\sqrt{\frac{1}{\langle a, a\rangle}}
$$

correspondente a uma solução da equação (3.6) para $\psi(\Delta J)=-\frac{a}{\langle a, a\rangle}$.

A margem de segurança $\mathcal{M}$, calculada através da equação (3.7), corresponde a uma solução de mínima norma.

A escolha da representação dada pela equação (3.4), tem sido importante, isto porque permite realizar as análises levando-se em conta a estrutura real do sistema (sem redução).

Definição 3.1. Autovalor crítico $\left(\lambda_{\min }\left(J^{0}\right)\right)$ é definido como sendo o menor autovalor positivo da matriz jacobiana $J^{0}$.

O autovalor crítico (definição 3.1) corresponde ao autovalor de interesse para análise da estabilidade de tensão, tendo em vista que é aquele que indica a máxima aproximação do colapso de tensão e, portanto, apresenta maior possibilidade de se tornar nulo à medida que houver o aumento da demanda de energia elétrica.

A partir da álgebra matricial, pode-se chegar ao seguinte resultado [5, 2]:

$$
\lambda_{\min }\left(J^{0}\right) \geq \mathcal{M} \geq 0 .
$$

Deste modo, a margem de segurança $\mathcal{M}$ representa uma estimativa pessimista (favorável à segurança do sistema) do parâmetro $\lambda_{\min }\left(J^{0}\right)$. A estimativa de $\lambda_{\min }\left(J^{0}\right)$ pode ser calculada, de forma alternativa, aplicando-se a norma matricial inversa de $J^{0}$. Este resultado é similar ao apresentado na referência [8]. O índice $\mathcal{M}$ será, então, usado como critério de inferência da estabilidade estática da tensão de sistemas elétricos de potência, via redes neurais, como formulado nesta seção.

\section{Rede Neural ARTMAP Fuzzy}

A rede neural ARTMAP Fuzzy [3] é formada por 2 módulos ART Fuzzy interligados através de um mecanismo chamado inter-ART, como será descrito adiante. Assim, primeiramente descreve-se o módulo ART Fuzzy. Para uma melhor fundamentação operacional das redes neurais ART-descendentes, os vetores usados são representados por linhas e não por colunas, como habitualmente é adotado na literatura especializada.

Os dados de entrada são denotados por vetor $a=\left[a_{1} \ldots a_{R}\right] R$-dimensional, e é normalizado com o intuito de evitar a proliferação de categorias. Assim, $\bar{a}=\frac{a}{|a|}$ sendo $\bar{a}$ o vetor de entrada normalizada e $|a|=\sum a_{i}$. 
O vetor de entrada será um vetor $2 R$-dimensional, denotado por:

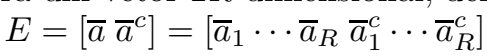

em que $\bar{a}^{c}=\bar{a}_{i}^{c}=1-\bar{a}_{i}^{c}$.

$\mathrm{O}$ vetor de atividade de $F_{2}$ é simbolizado por $y=\left[y_{1} \cdots y_{S}\right]$ sendo $S$ o número de categorias criadas em $F_{2}$. Deste modo, tem-se:

$$
y= \begin{cases}1, & \text { se o nó } J \text { de } F_{2} \text { é ativo } \\ 0, & \text { caso contrário }\end{cases}
$$

Os parâmetros utilizados no processamento da rede ART fuzzy são: parâmetro de escolha $(\eta>0)$, taxa de treinamento $(\beta \in[0,1])$ e parâmetro de vigilância $(\rho \in[0,1])$. Inicialmente todos os pesos possuem valor igual a 1 , indicando assim que não existe nenhuma categoria ativa.

A rede neural ARTMAP [3] é uma rede mais sofisticada, que emprega o treinamento supervisionado, i.e., baseado em estímulos de entrada-saída. É composta por dois módulos ART fuzzy; $A R T_{a}$ e $A R T_{b}$ interconectados através de módulo de memória associativa inter-ART, $F^{a b}$, que possui um mecanismo auto-regulador denominado match tracking, que tem a incumbência de maximizar a generalização e minimizar o erro da rede.

$\mathrm{O}$ vetor de entrada da rede $A R T_{a}$ é representado pelo vetor $a=\left[a_{1} \ldots a_{R_{a}}\right]$, $R_{a}$-dimensional, e o vetor de entrada da rede $A R T_{b}$ (que corresponde à saída desejada, referente ao padrão de entrada da rede $A R T_{a}$ ) é representado pelo vetor $b=\left[b_{1} \ldots b_{R_{b}}\right], R_{b}$-dimensional [3].

Os parâmetros usados na rede ARTMAP fuzzy são os mesmos usados na rede ART fuzzy. A diferença da rede neural ART fuzzy é o parâmetro de vigilância do módulo inter-ART, $\rho_{a b}\left(\rho_{a b} \in[0,1]\right)$.

A rede ARTMAP realiza o processamento de duas redes ART $\left(A R T_{a}\right.$ e $\left.A R T_{b}\right)$, sendo que, após a ressonância ser confirmada em cada rede, tem-se $C$ categoria ativa para a rede $A R T_{a}$ e $K$ categorias ativas para a rede $A R T_{b}$.

Através do processo match tracking é verificado se a categoria ativa no $A R T_{a}$ corresponde ao vetor de saída desejado apresentado no $A R T_{b}$. O critério de vigilância é dado por [3]:

$$
\frac{\left|y^{b} \wedge w_{C k}^{a b}\right|}{\left|y^{b}\right|} \geq \rho_{a b}
$$

em que $y^{b}$ é o vetor saída do $A R T_{b}$ (padrão de atividade $F_{2}^{b}$ ).

Se (4.1) não for satisfeito, é realizado um incremento mínimo no parâmetro de vigilância $A R T_{a}$, suficiente apenas para excluir a atual categoria e selecionar outra categoria que se tornará ativa e entrará novamente no processo até que (4.1) seja obedecida. Com a ressonância confirmada, os pesos dos módulos $A R T_{a}$ e $A R T_{b}$, são atualizados utilizando o mesmo critério da rede neural ART fuzzy. A adaptação para o módulo inter-ART é efetuada da seguinte forma: $w_{C k}^{a b}=1$ para $k=K$ e $w_{C k}^{a b}=0$ para $k \neq K$.

\subsection{Estímulo de Entrada da Rede Neural ARTMAP Fuzzy}

Os vetores padrões de entrada da rede neural são definidos como [4]:

$$
X=[P Q Z]
$$


sendo: $X$ o vetor padrão de entrada da rede neural; $P=\left[\begin{array}{llll}P_{1} & P_{2} & \cdots & P_{n}\end{array}\right] ; \quad Q=$ $\left[Q_{1} Q_{2} \cdots Q_{n}\right] ; P_{i}$ a potência ativa da i-ésima barra do sistema; $Q_{i}$ a potência reativa da i-ésima barra do sistema; $Z$ o vetor contendo as informações em código binário e, $n$ o número de barras do sistema.

Para a realização do treinamento - extração do conhecimento baseado em estímulos de entrada / saída - deve-se proceder a apresentação de um conjunto de dados, no caso, $X=\left[\begin{array}{lll}P & Q\end{array}\right]$ (entrada) e Y (saída), constituindo um conjunto de pares de treinamento. Trata-se de geração por vetores $P$ e $Q$, para a geração e para a carga do sistema por um procedimento de distribuição aleatória da geração (despacho aleatório para atender a demanda) e, também, de distribuição aleatória da carga (demanda aleatória) [4]. O vetor Z é formado por informações (em código binário), representando a topologia da rede elétrica, os dados das contingências, etc.

Para se gerar um grande espectro de variação da demanda, o critério a ser usado refere-se à distribuição aleatória da demanda e, conseqüentemente, da geração para atender a demanda, tomando-se variações percentuais da carga e da geração em torno do caso base (considerando como sendo o perfil de geração / carga de 100\%).

\subsection{Estímulo de Saída da Rede Neural ARTMAP Fuzzy}

Os estímulos de saída compreendem, neste artigo, os valores da margem de segurança $(\mathcal{M})$, calculados conforme descrito na Seção 3. Ou seja, os padrões de treinamento correspondem aos parâmetros: $X_{j}=\left[\begin{array}{lll}P_{j} & Q_{j} & Z_{j}\end{array}\right]$ (entradas); $Y_{j}=\left[\mathcal{M}_{\mathrm{j}}\right]$ (saidas); $j=1,2, \cdots, n p$, sendo $\mathcal{M}_{\mathrm{j}}$ a $j$-ésima margem de segurança representada por classes de severidade; $\overline{n p}$ o número de pares de padrões para a fase de treinamento.

A saída $\mathcal{M}_{\mathrm{j}}$ é codificada por classes (em código binário), sendo que cada classe expressa o grau de severidade.

\section{Aplicação}

Apresentam-se, a seguir, os resultados obtidos através do uso do método proposto, considerando-se um sistema composto por 45 barras, 72 linhas de transmissão e 10 máquinas síncronas. Os dados das máquinas síncronas e do sistema de transmissão pode ser visto na Figura 2.

O treinamento da rede neural foi realizado considerando-se um conjunto de 700 perfis de geração / carga e respectivas margens de segurança $\mathcal{M}$. Cada perfil corresponde a um redespacho de geração em relação ao caso base, realizado de forma aleatória para atender a demanda, também fixada de modo aleatório em cada barra. O universo de variação de carga encontra-se correspondido entre 65 e 135\%, em relação à carga total do sistema. Portanto, cada perfil é gerado considerando-se um percentual de variação em torno do estado nominal (caso base) e determinada semente para o processo de geração de seqüências aleatórias. Assim, para um mesmo percentual, diferentes sementes poderão gerar diferentes despachos de geração de diferentes perfis de carga. Realizado o treinamento da rede neural, os testes (análise da estabilidade de tensão) podem ser efetivados, cujos resultados são mostrados na Tabela 1. Os resultados da análise são apresentados na forma de classes de 1 a 10. 


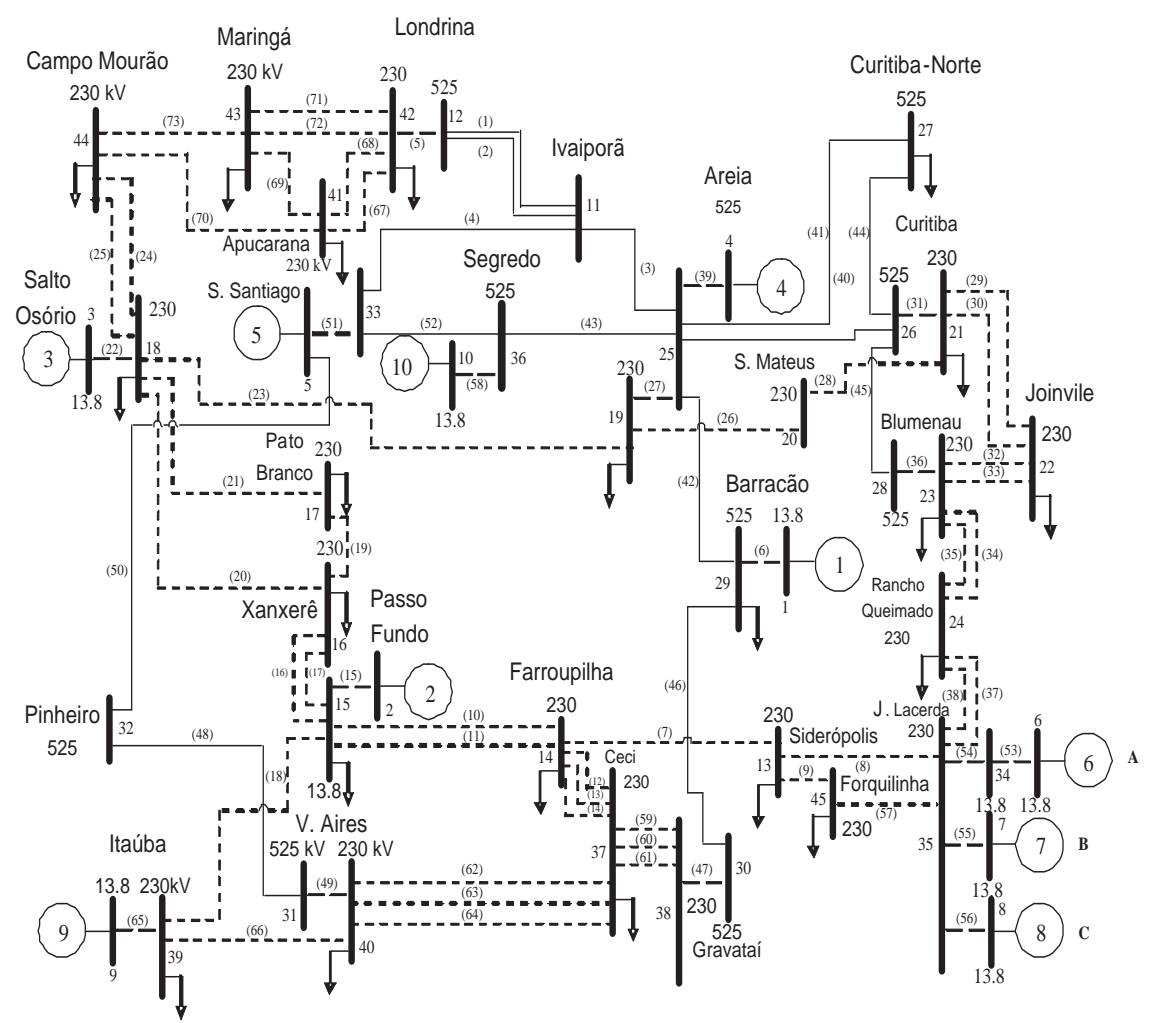

(--) Numero de linhas de transmissão.

Figura 2: Representação do sistema teste.

Estas classes são adotadas visando constituir as saídas em código binário, i.e., um modo mais adequado para se usar as redes neurais da família ART. Assim, adotou-se a seguinte definição das classes $1,2, \ldots, 10$ (representação com 4 bits) correspondem a $0 \leq \mathcal{M} \leq 0.1,0.1 \leq \mathcal{M} \leq 0.2, \ldots, 0,9 \leq \mathcal{M} \leq 1.0$, respectivamente. Com estas definições, pode-se inferir sobre as "distâncias" que cada perfil de geração / carga se encontra em relação à fronteira da establidade de tensão. Tais intervalos, associados às classes, podem ser definidos, aumentando-os ou diminuindo-os de acordo com o interesse do usuário. Neste caso, devendo-se ajustar o número de bits em função da maior ou da menor discretização usada. Ressalta-se que os acertos do sistema neural são superiores a 85\%, considerando-se 700 simulações realizadas. Este percentual, certamente irá aumentar, à medida em que novos padrões forem incorporados à base de dados, através do processo referente ao treinamento continuado. 
Tabela 1: Comparação dos métodos.

\begin{tabular}{|r|c|c|c||c|c|c|c|}
\hline & & \multicolumn{2}{|c|}{ Margem $\mathcal{M}$} & & & \multicolumn{2}{c|}{ Margem $\mathcal{M}$} \\
\hline$\%$ & Semente & Simul & $\begin{array}{c}\text { Artmap } \\
\text { Fuzzy }\end{array}$ & $\%$ & Semente & Simul & $\begin{array}{c}\text { Artmap } \\
\text { Fuzzy }\end{array}$ \\
\hline \hline-32.5 & 123 & .5994 & 6 & 17.5 & 123 & .6722 & 7 \\
\hline-27.5 & 123 & .6354 & 7 & 17.5 & 6691 & .6183 & 7 \\
\hline-22.5 & 614 & .7096 & 8 & 22.5 & 145 & .6055 & 6 \\
\hline-12.5 & 31 & .6551 & 8 & 22.5 & 221 & .6528 & 7 \\
\hline-7.5 & 123 & .7003 & 7 & 22.5 & 377 & .5662 & 6 \\
\hline-2.5 & 7116 & .7134 & 8 & 27.5 & 7116 & .4349 & 4 \\
\hline 7.5 & 31 & .7089 & 8 & 27.5 & 8555 & .5840 & 6 \\
\hline 12.5 & 74 & .6694 & 7 & 32.5 & 123 & .5873 & 6 \\
\hline
\end{tabular}

\section{Conclusão}

Foi apresentada uma nova metodologia para análise de estabilidade de tensão estática de sitemas elétricos de potência multinodal. Trata-se de uma metodologia usando uma rede neural ARTMAP fuzzy, cujo treinamento é realizado a partir de uma base de dados gerada via simulação (realizada usando o programa computacional Simul [4]); cálculo de fluxo de potência, bem como outras grandezas que foram implementadas visando atender a necessidade do estudo proposto. Tendo em vista que a estratégia de obtenção desta base de dados (definição de perfil de geração e de carga de modo aleatório) permitiu estabelecer um espectro de variação de modos operativos do sistema que "simule" estados operativos reais, a arquitetura ARTMAP fuzzy, tal como as demais redes da família ART, apresenta a característica de plasticidade que admite implementar o treinamento continuado, o que se diferencia, de forma vantajosa, em relação às tradicionais redes neurais comumente usadas na literatura especializada.

Abstract. This work presents a new methodology for the analysis of the static voltage stability in electric power systems. It is a neural inference system based on fuzzy ARTMAP architecture. This system presents accurate results with very fast response, being able to realize real time analysis. Besides, it allows the users work with flexibility where structural modifications are required (real situation in system operation), when compared to tradicional neural networks used in specialized literature. To illustrate the proposed neural network an apllication is presented considering an eletrical power system composed by 45 buses, 72 transmission line and 10 syncrhonous machines.

\section{Referências}

[1] L.D. Arya, S.C. Choube, M. Shrivastava, Technique for voltage stability assessment using newly developed line voltage stabiblity index, Energy Conversion and Management, 49, No. 2 (2008), 267-275. 
[2] S. Barnett, C. Storey, "Matrix Methods in Stability Theory", Thomas Nelson and Sons Ltd, London, 1970.

[3] G.A. Carpenter, S. Grossberg, N. Markuzon, J.H. Reynolds, D.B. Rosen, Fuzzy ARTMAP: A neural network architecture for incremental supervised learning of analog multidimentional maps, IEEE Transactions on Neural Networks, $\mathbf{3}$, No. 5 (1992), 698-713.

[4] W.P. Ferreira, M.C.G. Silveira, A.D.P. Lotufo, C.R. Minussi, Transient stability analysis of electric energy system via a Fuzzy ART-ARTMAP neural network, Electric Power System Research, 76 (1996), 475-484.

[5] J.C. Geromel, "Methods and Techniques For Decentralized Control Systems Analysis and Design", Cooperativa Libraria Universitaria del Politecnico, Milano, 1987.

[6] S. Haykin, "Neural Networks: A Comprehensive Foundation", Prentice-Hall, New Jersey, 1994.

[7] C.-T Su, F.C. Chang, Quick evaluation of determinants, Applied Mathematics and Computation, 75 (1996), 117-118.

[8] A. Tiranuchit, R.J. Thomas, A posturing strategy against voltage instabilities in electric power systems, IEEE Transaction on Power Systems, 3, No. 1 (1988), 87-93. 